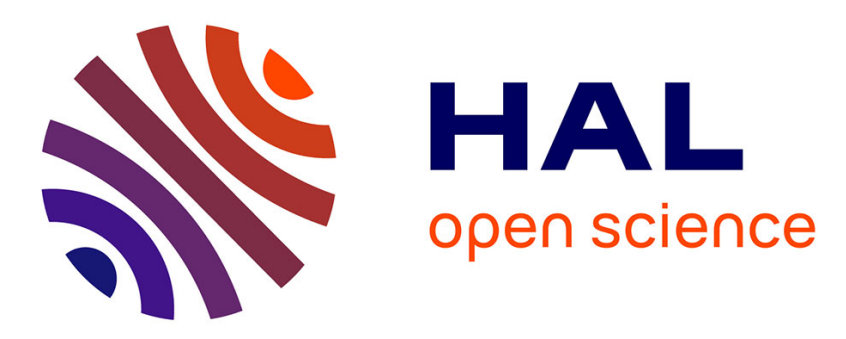

\title{
Milieux, hommes et techniques dans la vallée de la Kolimbiné (bassin supérieur du Sénégal, mali)
}

Christian Dupuy

\section{To cite this version:}

Christian Dupuy. Milieux, hommes et techniques dans la vallée de la Kolimbiné (bassin supérieur du Sénégal, mali) : Premier bilan d'un inventaire archéologique. Les Nouvelles de l'archéologie, 2010, 120-121, pp.81-86. halshs-00686800

\section{HAL Id: halshs-00686800 \\ https://shs.hal.science/halshs-00686800}

Submitted on 11 Apr 2012

HAL is a multi-disciplinary open access archive for the deposit and dissemination of scientific research documents, whether they are published or not. The documents may come from teaching and research institutions in France or abroad, or from public or private research centers.
L'archive ouverte pluridisciplinaire HAL, est destinée au dépôt et à la diffusion de documents scientifiques de niveau recherche, publiés ou non, émanant des établissements d'enseignement et de recherche français ou étrangers, des laboratoires publics ou privés. 


\section{Milieux, hommes et techniques dans la vallée de la Kolimbiné (bassin supérieur du Sénégal, Mali)}

Premier bilan d'un inventaire archéologique

Christian Dupuy*

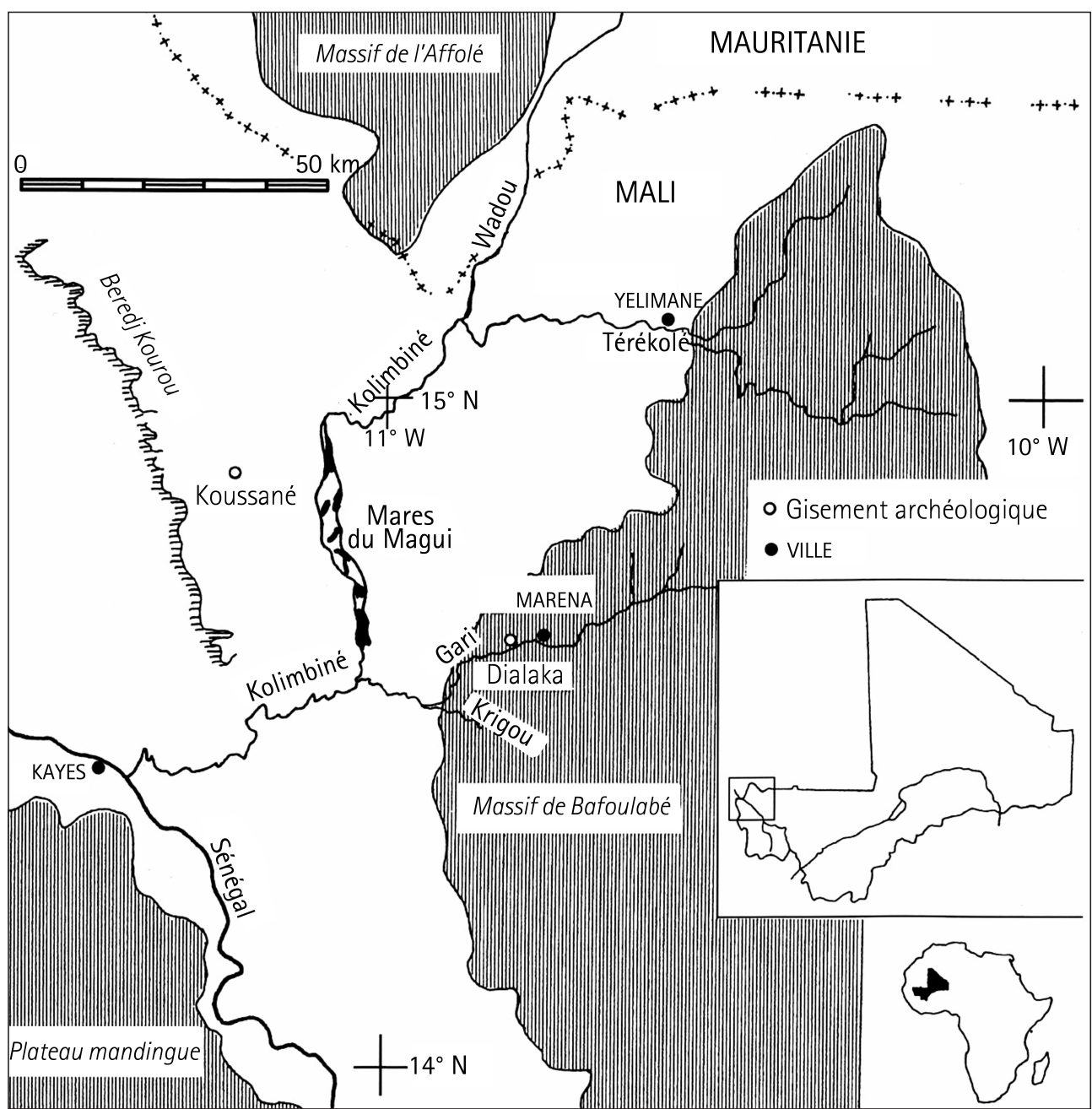

Fig. 1 - Situation géographique de la vallée de la Kolimbiné, un affluent de la rive droite du Sénégal à la hauteur de Kayes (Mali)

\section{Historique et contexte des recherches}

\section{* UMR 5060 CNRS, Belfort; \\ Universités tous âges, Lyon, dupuy.family-christian@wanadoo.fr}

\begin{abstract}
u début des années 1990, D. Commelin, O. Dutour, M. Raimbault et moi-même Aenvisagions de mener des fouilles dans l'Adrar des Iforas en des lieux a priori favorables à l'établissement de relations entre art rupestre, habitats et sépultures. L'objectif était d'apporter des éléments de réponse aux nombreuses questions soulevées dans les années 1980 sur le peuplement du Sahara malien durant l'Holocène. Malheureusement, la région devint inaccessible en raison de la rébellion armée des Touaregs contre le pouvoir central. L'Institut des sciences humaines de Bamako me
\end{abstract}




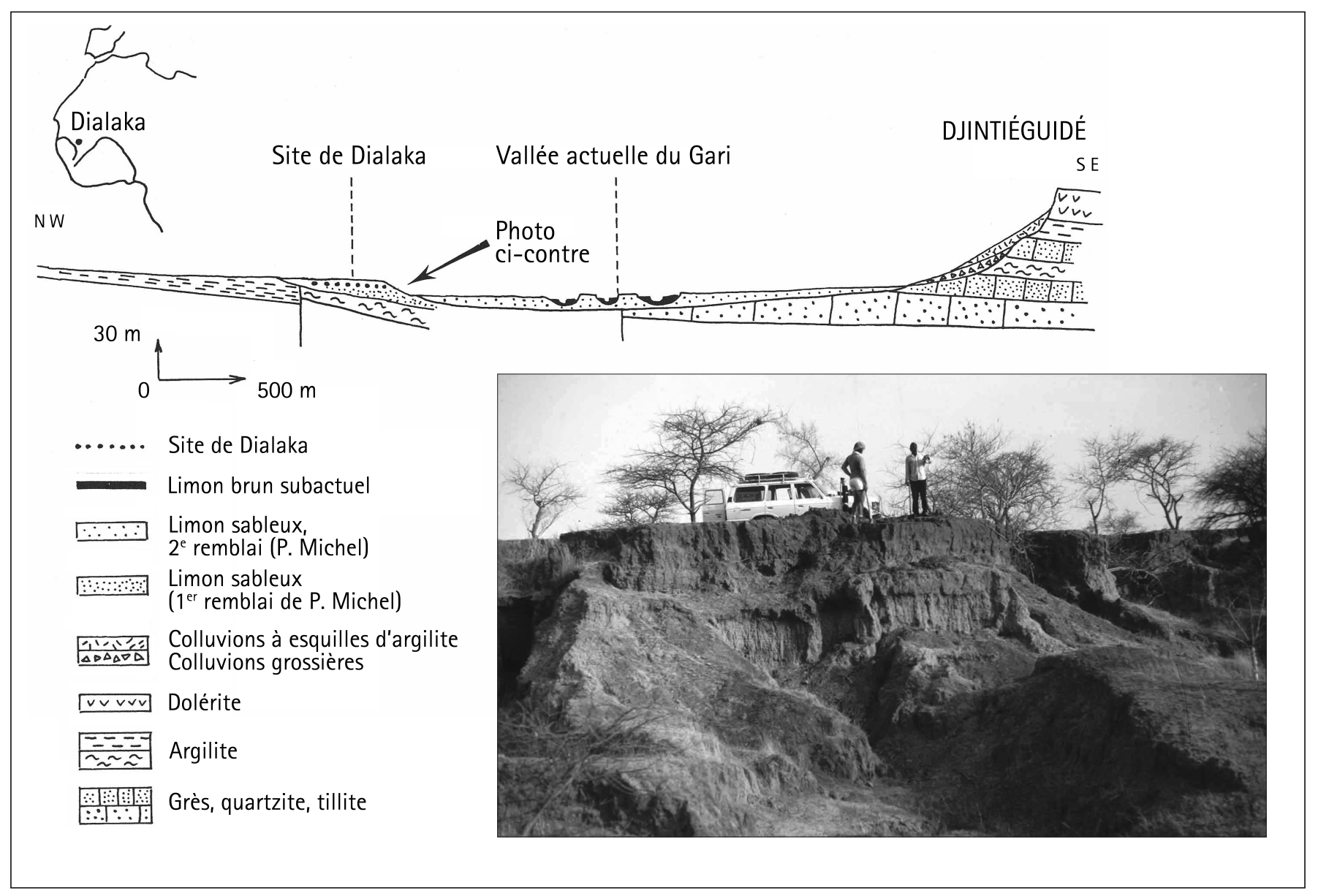

Fig. 2 - Position du site archéologique de Dialaka dans la vallée du Gari, au débouché du massif de Bafoulabé. Vue de la coupe sur laquelle on suit le niveau archéologique pendant plusieurs kilomètres et pratiquement sans interruption.

confia alors la responsabilité de l'inventaire archéologique dans la vallée de la Kolimbiné, un affluent de la rive droite du Sénégal à la hauteur de Kayes, qui n'avait jamais fait l'objet de prospection (fig. 1). Les recherches interdisciplinaires que je coordonnais participaient de deux programmes :

- le premier, intitulé "Métallurgies africaines" (METAF), était international; il était supervisé par Pierre de Maret de l'Université libre de Bruxelles et financé par l'Agence de coopération culturelle et technique (AсcT). En 1995, les prospections réalisées dans ce cadre, révélèrent la richesse de la vallée en vestiges sidérurgiques;

- le second, intitulé "Dynamique et évolution du peuplement dans les vallées du Niger et du Sénégal depuis le Pléistocène final", était conçu pour durer deux ans avec possibilité de renouvellement, suivant un protocole de coopération établi entre l'Institut des sciences humaines de Bamako et l'université d'Aix-Marseille-1 que le ministère de la Coopération française s'engageait à financer. Plusieurs sondages furent effectués en 1996 sur des habitats stratifiés : deux ateliers sidérurgiques, un tumulus et une butte de terre recouverte d'une imposante accumulation de tessons furent fouillés pendant que se déroulaient des enquêtes sur la céramique traditionnelle et que se multipliaient les observations sur le paysage. Les campagnes de fouilles qui devaient suivre n'ont jamais eu lieu, faute de financement et malgré nos relances répétées auprès du ministère de la Coopération française. Cette absence de suivi programmatique à la fin des années 1990, très dommageable sur le plan scientifique et ô combien décevante pour les chercheurs impliqués, a plusieurs causes. Elle tient d'abord, et peut-être surtout, à l'intégration du ministère de la Coopération dans le ministère des Affaires étrangères et aux restructurations administratives subséquentes de ses services; elle tient aussi et simultanément, aux changements successifs des responsables administratifs, liés au rythme soutenu des alternances politiques; elle tient enfin, à l'élaboration de projets archéologiques nouveaux probablement perçus comme concurrents du nôtre (il aurait été plus juste d'y voir des projets complémentaires) qui, eux, ont bénéficié de financements. Le ministère de la Coopération française n'a jamais justifié sa décision de ne plus soutenir notre programme. Comment interpréter ce silence? Quelles évaluations scientifiques ont été conduites et, s'il y en eut, par qui l'ont-elles été? En plus de la frustration engendrée par l'absence de réponse à ces questions, l'espoir 
d'obtenir un poste de chercheur s'est amenuisé au fil des ans, faute de perspective professionnelle en archéologie africaine tant au CNRS qu'à l'IRD ou à l'Université.

En 1998, la Fondation Singer-Polignac m'attribua une bourse pour étudier les vestiges métallurgiques découverts en 1996. Depuis 1999, les conférences que je donne dans les universités tous âges de Lyon sur "L'art et l'histoire des civilisations africaines" attirent régulièrement des dizaines d'auditeurs dont la curiosité et la fidélité m'encouragent à persévérer dans mes recherches. Jusqu'à présent, j'ai publié six articles consacrés à l'analyse des vestiges mis au jour lors des sondages et des fouilles menés dans la vallée de la Kolimbiné. De nombreuses données restent à exploiter, y compris celles recueillies au cours des enquêtes sur la poterie traditionnelle de la région, avant de pouvoir proposer une monographie archéologique et ethnoarchéologique sur ce secteur.

\section{Bilan scientifique intermédiaire}

Paysages et peuplement L'existence de deux surfaces d'érosion distinctes au débouché du massif de Bafoulabé, toutes deux situées plusieurs mètres au dessus des lits actuels des cours d'eau, pose la question de l'évolution du paysage en rapport avec les fluctuations climatiques du Pléistocène et de l'Holocène et l'anthropisation.

Les âges proposés pour ces formations sédimentaires à base de sable et de silt - Pléistocène terminal pour la plus haute surface d'érosion et Holocène pour la plus basse - l'ont été, il y a plusieurs dizaines d'années, sur la base de corrélations avec d'autres régions de l'Afrique nord-tropicale (Michel 1960 et 1973). Des études récentes montrent que l'essentiel des dépôts sableux éoliens en milieu sahélien est en place depuis très longtemps, souvent depuis le début du Pléistocène (Durand 1995). Selon nous, les variations climatiques quaternaires ont seulement provoqué le remaniement périodique, par l'eau et par le vent, de ces dépôts anciens auxquels de nouveaux se sont mêlés au fil du temps. Une estimation de l'importance de cette sédimentation est fournie dans la vallée de la Kolimbiné par les sites de Dialaka et de Koussané datés de l'âge du Fer ancien. En deux mille ans, ils ont été recouverts par un sol à base de sable et de silt d'une puissance de 0,3 à 0,8 mètres (Dupuy, Riser \&t Sissoko 1995; Dupuy et al. 2001-2002). Sédimentation et remaniements perpétuels, caractéristiques essentielles de toute formation superficielle, effacent progressivement les formes anciennes. Ce mécanisme pourrait expliquer l'absence apparente dans les vallées prospectées de formations quaternaires anciennes. Cette expression "absence apparente» est employée volontairement car on peut supposer que de telles formations existent sans avoir pu être reconnues avec certitude. Les coupes permettant d'observer la base des dépôts sédimentaires dans les vallées n'en ont pas montré jusqu'à présent. Par contre, une coupe en position haute, vers la source du Gari au débouché des plateaux mandingues, a livré en stratigraphie quelques outils sur quartzite qui pourraient être paléolithiques.

Comme indiqué plus haut, deux sites archéologiques en stratigraphie ont été repérés et sondés. À Dialaka, les vestiges sont situés entre 0,3 et 0,8 mètres sous la plus haute surface d'érosion qui domine de plusieurs mètres le niveau des villages actuels, où les eaux souterraines sont atteintes par des puits de plus de dix mètres de profondeur (fig. 2). L'occupation est datée au ${ }^{14} \mathrm{C} d u$ $\mathrm{I}^{\mathrm{er}}$ millénaire avant J.-C. Son abandon semble remonter aux premiers siècles de l'ère chrétienne. Si l'aridité marquée à cette époque dans le bassin du Sénégal a probablement favorisé cet abandon et causé les dépôts postérieurs de sédiments, le fait que les villages soient aujourd'hui installés plusieurs mètres en contrebas se justifie par une baisse du niveau des nappes phréatiques. Nos observations de terrain et la comparaison sur photographies aériennes de la morphologie actuelle de trois vallées à densité et nature de peuplement différentes montrent que les déboisements et les défrichements en relation avec les activités agricoles conditionnent pour une large part l'entaille de ces vallées, dont dépend le niveau des nappes phréatiques. Ces constats nous font lier l'abandon du site de hauteur de Dialaka, occupé au I ${ }^{\mathrm{er}}$ millénaire avant J.-C., à une redistribution des ressources en eau sous l'effet du creusement des vallées, par suite d'une aridité marquée et de pratiques culturales plus extensives, peut-être favorisées par la multiplication des outils et des instruments aratoires en fer et, simultanément, par un accroissement de la population.

Un bas fourneau de l'âge ancien du Fer Un décapage horizontal de 35 mètres carrés sur le gisement archéologique de Koussané a permis la mise au jour de vestiges sidérurgiques en relation avec une opération de réduction directe d'un minerai de fer siliceux. Deux fragments de charbon de bois inclus dans la scorie du fond de four ont donné comme âge ${ }^{14} \mathrm{C}: 1715 \pm 55 \mathrm{BP}$. Cette date s'accorde avec la chronostratigraphie du gisement. Il s'agit de la plus ancienne réduction directe actuellement connue dans le bassin hydrographique du Sénégal (Dupuy \&t Rolando 1999).

Ce bas fourneau de l'âge du Fer ancien se composait d'une fosse en cuvette au-dessus de laquelle était construite une cellule ou colonne à base de boudins d'argile superposés qui recevait au moins quatre tuyères en pisé. L'une d'elles, disposée obliquement à travers le corps du four, a favorisé la formation d'une éponge de fer en partie haute de la scorie comme le montrent les examens métallographiques (fig.3). À la fin de la réduction, la construction en élévation a été déplacée de deux à trois mètres. Dès lors à ciel ouvert, le bloc de 90 kilos de scorie qui s'était formé dans le fond de four a été brisé et disloqué aux deux tiers pour permettre le prélèvement de l'éponge de fer. Les résultats des analyses chimiques effectuées sur le minerai et la scorie permettent d'estimer autour de 11,5 kilogrammes la masse de métal brut produit (fig. 4 et Dupuy et al. 2001-2002 : 77-84).

\section{Trois inhumations antéislamiques} sous un tumulus de pierre à Dialaka (fig. 5)

La fouille de ce tumulus à dépression sommitale avait plusieurs objectifs : nous souhaitions vérifier que ce type de monument fréquent dans la région avait une fonction funéraire, préciser le rituel d'inhumation à partir des ossements et du mobilier d'accompagnement susceptibles d'être mis au 
jour, déterminer l'âge de ces vestiges et, pour finir, nous interroger sur leur relation avec les habitats en contrebas occupés durant le I ${ }^{\mathrm{er}}$ millénaire avant J.-C.

La fonction funéraire présumée du monument a été confirmée par la découverte de trois squelettes en très mauvais état de conservation, sans matériel associé ou du moins préservé. La répartition des ossements à la surface du sol ainsi que les contacts avec superposition en impliquant cinq plaident en faveur d'un rituel funéraire que l'on peut résumer de la manière suivante :

1 - dépôt simultané en décubitus dorsal des cadavres de deux jeunes adultes de stature assez élevée, décédés selon des circonstances indéterminables ;

2 - dépôt désordonné au-dessus de ces individus des restes osseux d'une troisième personne de stature inférieure. Il s'agit très probablement d'une inhumation secondaire;

3 - recouvrement de l'ensemble sépulcral par un imposant amas de pierres prélevées sur place dans les éboulis de pente;
4 - aménagement d'une dépression au sommet de la construction.

La bioapatite tirée des ossements a fourni la date ${ }^{14} \mathrm{C}$ de $1515 \pm 60$ BP. Loin de combler les énormes lacunes des connaissances dans le domaine de l'archéologie funéraire ouestafricaine, l'âge obtenu à Dialaka fournit un nouveau jalon chronologique en ce qui concerne la tradition funéraire de l'inhumation simultanée de plusieurs individus en décubitus dorsal sous des constructions monumentales (Dupuy et al. 2006).

\section{Diversité du peuplement et unité typologique des jarres à eau}

Quatre communautés distinctes, tant par la langue que par les traditions culturelles, sont représentées dans la vallée de la Kolimbiné : des Soninkés et des Khassonkés sédentaires, des Peuls et des Maures, sédentaires ou nomades. La diversité architecturale des habitations témoigne de cette pluralité du peuplement. Les matériaux de construction - terre, bois, fibres végé-

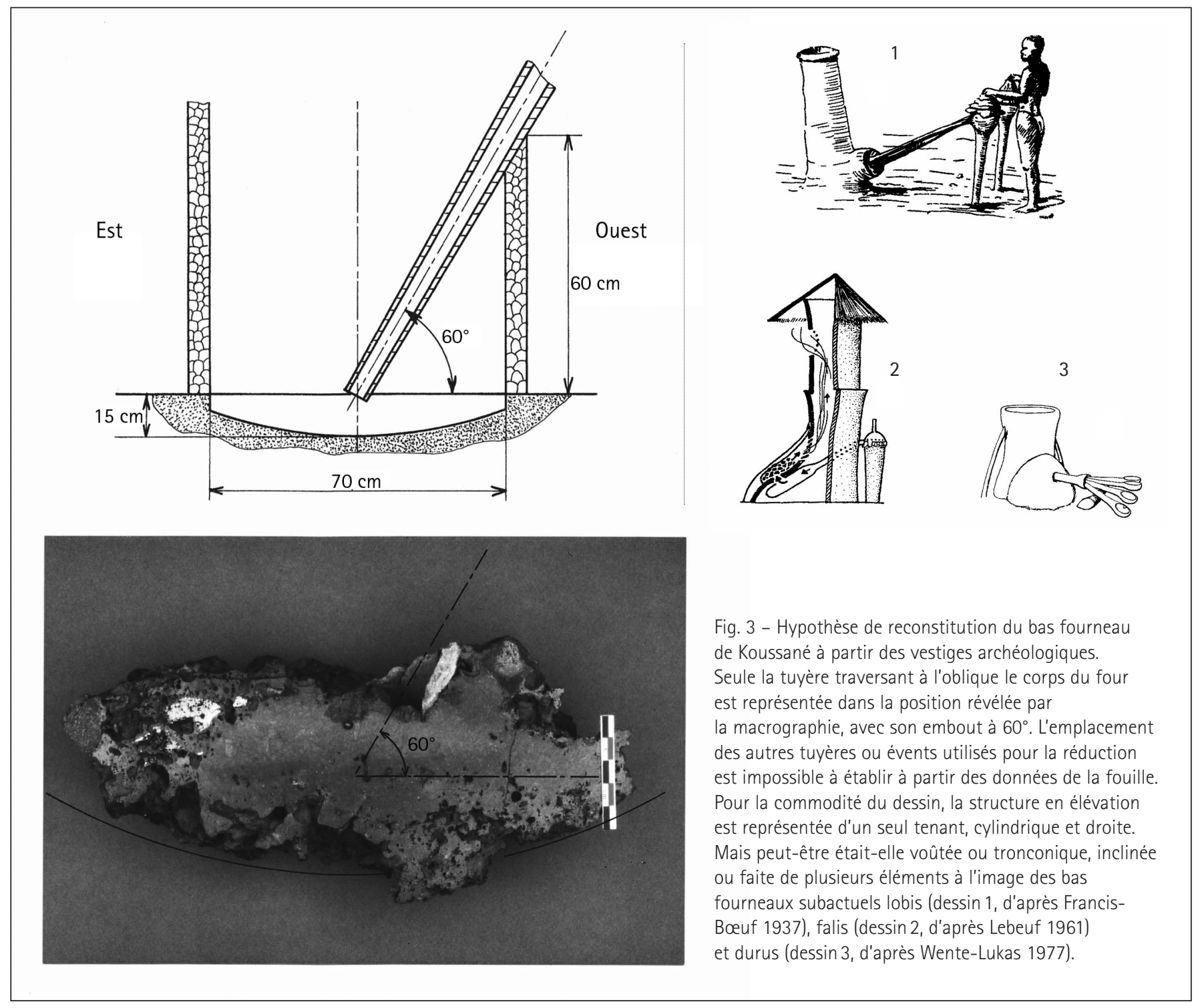


Fig. 4 - Bilan matière simplifié de la réduction, établi à partir des résultats des analyses chimiques par spectrométrie d'émission

réalisées sur le minerai et la scorie du fond du four

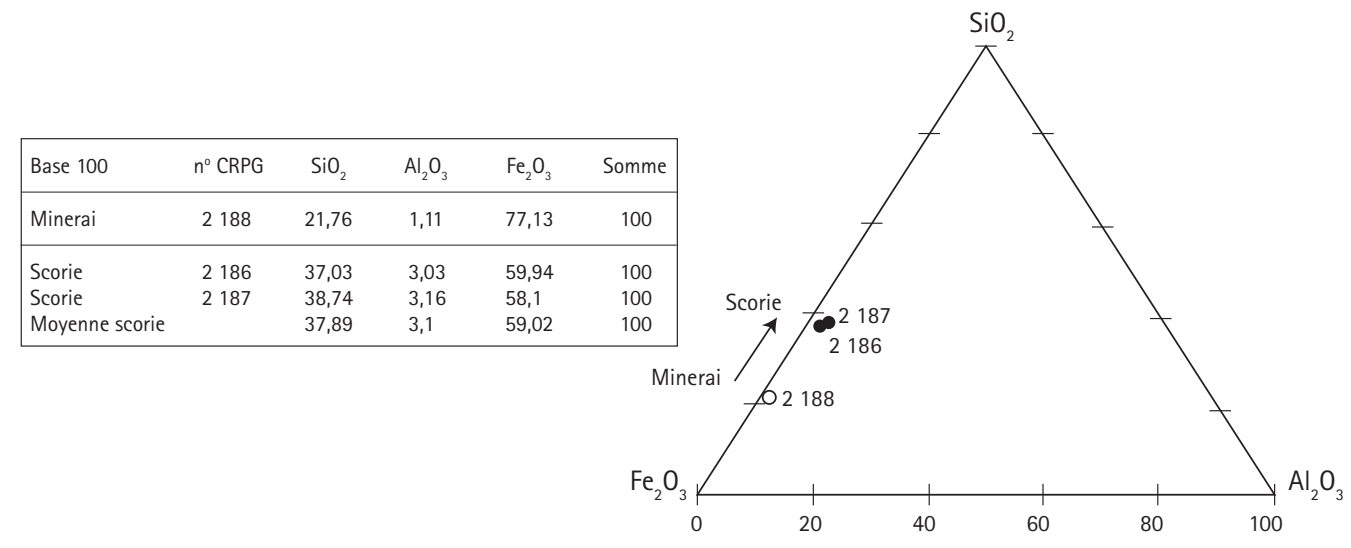

\begin{tabular}{|c|c|c|c|c|}
\hline & $\mathrm{Si}$ & $\mathrm{Al}$ & $\mathrm{Fe}$ & BILAN MATIĖRE DE LA RÉDUCTION \\
\hline $100 \mathrm{~g}$ minerai & 10,22 & 0,59 & 54 & $54 \mathrm{~g}$ de fer dans minerai donnent \\
\hline $100 \mathrm{~g}$ scorie (moy. scorie) & 17,8 & 1,64 & 41,31 & $41,31 \mathrm{~g}$ de fer dans scorie $+12,69 \mathrm{~g}$ fer-métal \\
\hline Différence & 7,58 & 1,05 & $-12,69$ & Estimation de la masse de scorie produite dans le bas fourneau : $90 \mathrm{~kg}$ \\
\hline & & & & $\begin{array}{l}12,69 \mathrm{~g} \text { de fer-métal poue } 100 \mathrm{~g} \text { de scorie produits } \\
11,5 \mathrm{~kg} \text { de fer-métal pour } 90 \mathrm{~kg} \text { de scorie produits }\end{array}$ \\
\hline
\end{tabular}

tales - sont fragiles et se dégradent vite après l'abandon des maisons. Il nous a donc paru intéressant de voir si, en dehors de l'habitat, la céramique qui, elle, se conserve bien, pourrait permettre d'identifier les différents groupes en présence.

Seules les femmes soninkés et khassonkés, pour la plupart épouses de forgerons, fabriquent des poteries pendant la saison sèche. Ni les techniques de modelage de l'argile, ni les décors appliqués, ni les formes des vases, ni le mode de cuisson - en tas à même le sol et à ciel ouvert - ne permettent d'individualiser les productions.

$\mathrm{Au}$ début de la saison des pluies, alors que les terres autour des villages sont ensemencées en mil, sorgho, maïs et arachide, certains agriculteurs soninkés quittent leur village pour des hameaux de cultures situés sur les plateaux mandingues ou au pied des escarpements. Ils en reviennent après les récoltes en laissant sur place, à l'intérieur des huttes, des objets usuels telles des jarres à eau qui serviront l'année suivante.

Les Peuls et les Maures sédentaires ne sont pas producteurs de céramiques mais ils utilisent quotidiennement des récipients en terre pour cuisiner, boire et stocker diverses denrées. Ils s'approvisionnent auprès des Soninkés et Khassonkés qui fabriquent des pots en excès destinés à la vente, à moins que des familles de forgerons soninkés dont les femmes sont potières ne soient établies dans leur village.

Durant la saison sèche, des nomades issus des régions arides plus septentrionales, à savoir des Peuls éleveurs de taurins et des Maures éleveurs de chèvres et de moutons, élisent campement sur les terres moissonnées. Pour leurs besoins quotidiens en eau, les Maures utilisent des outres en peau de chèvre ou de mouton auxquelles les Peuls préfèrent des jarres à eau. Les familles en possèdent une, parfois deux, d'une contenance avoisinant les dix litres. Ces jarres achetées auprès des potières soninkés sont en moyenne deux fois moins volumineuses que celles utilisées par les sédentaires.
En cas de casse au cours de la transhumance d'hivernage sur les terres salées de Mauritanie, les femmes attendent la saison sèche pour les remplacer par de nouvelles poteries achetées dans la vallée de la Kolimbiné.

Il découle de cette organisation que des jarres à eau de même type sont utilisées par les nomades et par les sédentaires, que ces derniers fabriquent ou non des poteries. Cette situation montre combien il peut s'avérer délicat d'évaluer, à partir du seul document céramique et ici en particulier à partir des jarres à eau, la diversité d'un peuplement dans la savane.

La céramique des Peuls

L'aire géographique occupée par les Peuls est très vaste; elle s'étend d'est en ouest, du bassin du lac Tchad à la bordure atlantique, et couvre la majeure partie du Sahel en mordant par endroit sur les zones forestières plus méridionales. Où qu'ils se trouvent, les Peuls élèvent des bovins (taurins, zébus et/ou sangas). Certains groupes sont nomades. D'autres ne sont mobiles qu'une partie de l'année. Ceux qui vivent à la fois de l'élevage et de l'agriculture sont sédentaires.

$\mathrm{Ni}$ les groupes nomades ni les groupes sédentaires ne produisent de la céramique dans la vallée de la Kolimbiné. Tous s'approvisionnent en poteries auprès des agriculteurs soninkés et khassonkés. En conséquence, leur céramique est semblable et son analyse comme vestige archéologique ne permettrait donc pas d'identifier leur présence ici.

Les enquêtes ethnoarchéologiques menées dans deux autres régions de l'Ouest africain auprès de groupes peuls sédentaires qui, eux, fabriquent des poteries montrent combien, même dans cette situation, il est difficile d'assigner au document céramique une valeur de marqueur culturel qui puisse servir à la reconstitution de l'histoire ancienne de ce peuple. La première de ces enquêtes a été menée en 1972 par N. David et $\mathrm{H}$. Hennig auprès des potières du village de Bé, dans le 


\section{Dossier La coopération archéologique française en Afrique, 1}

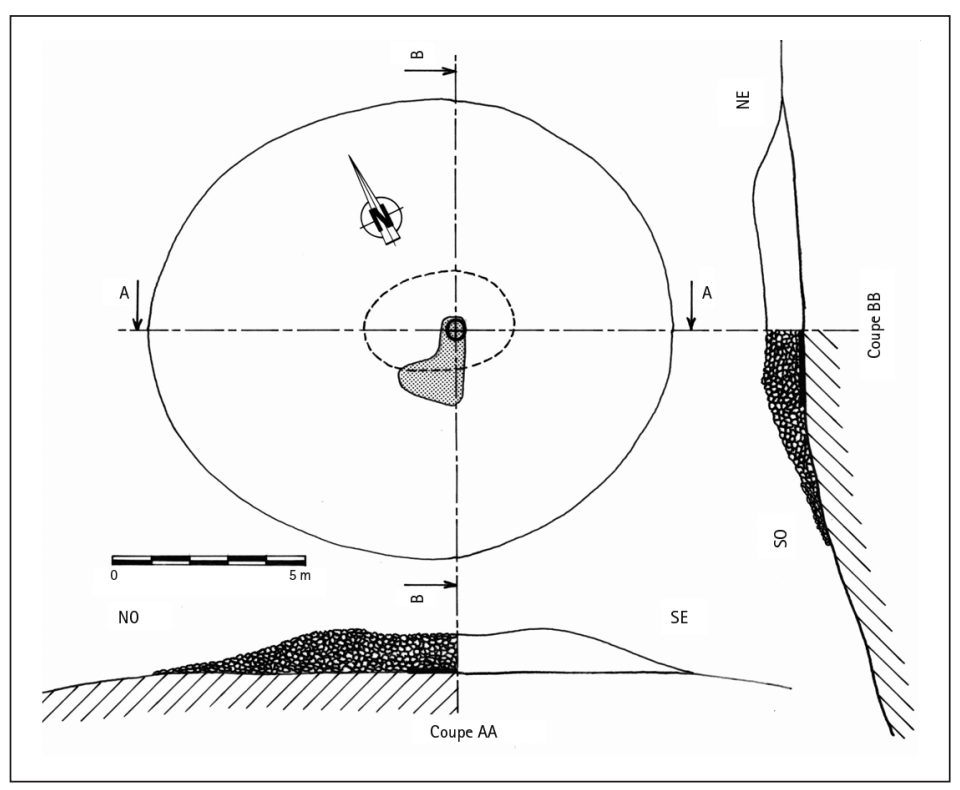

Fig. 5 - Plan général du tumulus fouillé à Dialaka. La dépression sommitale est délimitée par les pointillés. La zone couverte de points correspond à l'aire de répartition des ossements apparus sous l'amas pierrier au niveau du sol. Les axes du monument se recoupent au niveau du repère de fouille.

réponses à ces questions passent par l'établissement de typochronologies régionales aussi précises que possible, aujourd'hui encore trop rarissimes; tel est notre objectif dans la vallée de la Kolimbiné. Parallèlement, la prise en compte des traditions actuelles du peuplement dans ce secteur et, plus largement, en Afrique de l'Ouest, devrait permettre de mieux cerner les limites au-delà desquelles les interprétations culturelles, technologiques, socioéconomiques et ethnohistoriques à accorder aux vestiges archéologiques ne peuvent aller.

\section{Bibliographie}

nord du Cameroun. Ces femmes peules façonnent l'argile par martelage au percuteur de bois sur un moule convexe. La partie supérieure de leurs vases est montée aux colombins. Les décors à base d'impressions roulées et d'incisions appliquées sous les cols seraient d'inspiration lamé, un groupe tributaire des Peuls depuis au moins le XVIII ${ }^{\mathrm{e}}$ siècle. La seconde enquête s'est déroulée dans la boucle du Niger, une région où les Peuls sont organisés en une société hiérarchisée. La technique de façonnage la plus utilisée ici par les potières peules, pour la plupart femmes de tisserands, est celle du pilonnage dans une forme concave, un percuteur en terre cuite à la main. Seul le col est monté à l'aide d'un colombin. En raison de la faible épaisseur des parois obtenues, les corps des récipients ne peuvent recevoir que des décors peints. Quelques potières et potiers des ethnies voisines modèlent l'argile selon cette même technique qu'E. Huysecom (1991-1992) est conduit à supposer d'origine peule en raison des noms vernaculaires de racine peule généralement donnés aux percuteurs en terre cuite.

De ce qui précède, on peut retenir, d'une part, qu'il n'existe pas de corrélation entre la communauté peule et un type de céramique et, d'autre part, que le sens des emprunts techniques en ce qui concerne la fabrication de la poterie varie au sein de cette communauté selon les groupes et les régions.

\section{Perspectives de recherches}

Sur un plan général, nos travaux ne vont pas sans poser plusieurs questions d'ordre socioéconomique. À quand remonte par exemple, dans le sud du Sahara, la pratique de l'élevage par des éleveurs à la mobilité commandée par les points d'eau et les pâturages, ou bien celle de l'agriculture céréalière sous pluies de mousson par des communautés villageoises? La différenciation de ces modes de vie s'inscrit-elle dans le Néolithique ancien comme le suggère l'art rupestre à gravures naturalistes de l'Adrar des Iforas à propos du nomadisme pastoral (Dupuy ce volume : 29-37)? Dans quelles limites les vestiges archéologiques permettent-ils de retracer cette histoire? Les
DAVID, N. \& H. HenNIG. 1972. "The ethnography of pottery. A Fulani case in archaeological perspective", Module (Reading, MA, Addison Wesley), $21: 1-29$.

Dupuy, C., P. Fluzin, A. Ploquin, A. Durand \& C. Rolando. 2001-2002. "Nouvelles données sur l'âge ancien des métaux au Mali», Sahara, $13: 61-90$.

DUPUY, C., J. RISER \& F. SISSOKO. 1995. "L'abandon du site protohistorique de Dialaka (Mali) à l'Holocène supérieurn, Quaternaire, 6, 2 : 57-61.

DUPUY, C. Et C. RoLANDO. 1999. "Un four de métallurgie du fer en stratigraphie à Koussané (Mali) : fouille, anthracologie et datations sur charbons", in : J. EVIN, C. OBerLIN, J.-P. Daugas \& J.-F. Salles (éd.), ${ }^{14}$ C et archéologie. Paris, Société préhistorique française (Revue d'Archéométrie, suppl.; Mémoires de la Société préhistorique française, 26) : 381-385.

Dupuy, C., J. F. SAliege \& M. TAVIer. 2006. «Trois inhumations sous tumulus pierrier à Dialaka (Ouest malien, bassin supérieur du Sénégal) ", in : C. Descamps \& A. Camara, Senegalia. Études sur le patrimoine ouestafrician. Hommage à Guy Thilmans. Paris, SePIA Éditions : 239-249.

DURAND, A. 1995, "Sédiments quaternaires et changements climatiques au Sahel central (Niger et Tchad)", Africa Geoscience Review, 2, 3/4 : 323-614.

Francis-Boeuf, C. 1937. "L'industrie autochtone du fer en Afrique occidentale française", Bulletin du Comité d'études historiques et scientifiques de l'Afrique occidentale française, $20: 403-454$.

HUYSECOM, E. 1991-1992. "Les percuteurs d'argile : des outils de potières africaines utilisés de la Préhistoire à nos jours", Bulletin du Centre genevois d'anthropologie, $3: 71-98$.

LEBEUF, J.-P. 1961. L'habitation des Fali, montagnards du Cameroun septentrional. Paris, Hachette, 608 pages.

Michel, P. 1960. "Note sur l'évolution morphologique des vallées de la Kolimbiné, du Karakoro et du Sénégal dans la région de Kayes", Dakar, rapport du Bureau de recherches géologiques et minières, 18 pages.

Michel, P. 1973. Les bassins des fleuves Sénégal et Gambie. Étude géomorphologique. Paris, ORSTOM (Mémoires ORSTOM, 63), 3 tomes, 752 pages.

Wente-LUKAS, R. 1977. "Fer et forgerons au sud du lac Tchad (Cameroun, Nigeria)", Journal des africanistes, 47, 2 : 107-122. 